Vásquez Rizo, F. E. y Gabalán Coello, J. (2006). Percepciones estudiantiles y su influencia en la evaluación del profesorado. Un caso en la Universidad Autónoma de Occidente, Cali-Colombia.

RELIEVE, v. 12, n. 2, p. 219-245. http://www.uv.es/RELIEVE/v12n2/RELIEVEv12n2 3.htm

\title{
REIIदVE
}

Revista ELectrónica de Investigación

y EValuación Educativa

\section{PERCEPCIONES ESTUDIANTILES Y SU INFLUENCIA EN LA EVALUACIÓN DEL PROFESORADO. UN CASO EN LA UNIVERSIDAD AUTÓNOMA DE OCCIDENTE, CALI - COLOMBIA}

\section{[Student perceptions and their influence on the evaluation of the teaching staff. A case in Universidad Autónoma de Occidente, Cali - Colombia]}

por

$\underline{\text { Article record }}$

$\underline{\text { About authors }}$

$\underline{\text { HTML format }}$
Fredy Eduardo Vásquez Rizo (fvasquez@uao.edu.co) Jesús Gabalán Coello (jgabalan@uao.edu.co) $\underline{\text { Ficha del artículo }}$

$\underline{\text { Sobre los autores }}$

Formato HTML

\begin{abstract}
The investigation results are presented: "An investigation or research about the most relevant variables from the student's point of view which influence the general opinion about the teacher's performance in the Universidad Autonoma de Occidente". The problems about the influence from the students perception in evaluating their teachers is taken on from two complementary perspectives: The first constitutes the qualitative analysis which has as a support the group interviews realized in each one of the academic programs. The second involves the quantitative analysis based on multivariate statistic tools. These perspectives allow elaborating a model to establish the grade of relevance each one of the items used to evaluate the teacher has, in relation with the general grading that is assigned to his/her performance. Finally, the most important aspects are evidenced for the students at the moment of stating a valuable judgment over their teachers.
\end{abstract}

\section{Keywords}

Student Perceptions, Evaluation, Quantitative Analysis, Qualitative Analysis, Teacher Performance, University

\begin{abstract}
Resumen
Se presentan los resultados de la investigación: "Estudio sobre las variables más relevantes, desde el punto de vista estudiantil, que influyen en la opinión general sobre el desempeño de los docentes en la Universidad Autónoma de Occidente". Se aborda la problemática sobre la influencia que tienen las percepciones estudiantiles en la evaluación del profesorado desde dos perspectivas complementarias: La primera de ellas constituye el análisis cualitativo, que tiene como soporte entrevistas grupales llevadas a cabo con estudiantes de cada uno de los Programas Académicos. La segunda involucra el análisis cuantitativo, basándose en herramientas de estadística multivariada. Estas perspectivas permiten elaborar un modelo que establece el grado de relevancia que tiene cada uno de los ítems a través de los cuales se evalúa un profesor, en relación con la calificación general que se asigna a su desempeño. Finalmente, se evidencia qué aspectos son los más importantes, para los estudiantes, en el momento de emitir un juicio valorativo sobre sus docentes.
\end{abstract}

\section{Descriptores}

Percepciones Estudiantiles, Evaluación, Análisis Cuantitativo, Análisis Cualitativo, Desempeño Docente, Universidad 
Vásquez Rizo, F. E. y Gabalán Coello, J. (2006). Percepciones estudiantiles y su influencia en la evaluación del profesorado. Un caso en la Universidad Autónoma de Occidente, Cali-Colombia.

RELIEVE, v. 12, n. 2, p. 219-245. http://www.uv.es/RELIEVE/v12n2/RELIEVEv12n2 3.htm

\section{INTRODUCCIÓN}

Las Instituciones de Educación Superior, conscientes del compromiso que tienen con la calidad académica, han establecido una política de evaluación en la cual se involucran sus tres funciones sustantivas (docencia, investigación y extensión o proyección social).

La evaluación del desempeño docente se asume como un proceso permanente enmarcado dentro de una concepción de calidad de la educación, enfocado hacia el perfeccionamiento de la docencia en una institución educativa. La utilización de diversas fuentes de información (alumnos, colegas, jefe inmediato y el mismo docente) permiten identificar de una manera comprehensiva la labor del profesor y, a partir de ellas, establecer políticas de mejoramiento institucional.

Por ello, las Instituciones de Educación Superior, en general, y la Universidad Autónoma de Occidente, en particular, construye un estudio tendiente a determinar la relación que existe entre las variables de desempeño docente. Cada Institución define un conjunto de variables desde las cuales se analiza la evaluación del docente desde la perspectiva de los estudiantes.

La Universidad Autónoma de Occidente es una Institución de Educación Superior de carácter privado, cuya misión es la de integrar, con perspectiva internacional, las funciones sustantivas de docencia, investigación y proyección social para contribuir a la formación de personas con visión humanística, creativas y emprendedoras, a la generación de conocimiento y a la solución de problemas del entorno regional, nacional e internacional.

La Institución de la cual se extrajeron los datos para el presente análisis cuenta con un amplio número de profesores (Tiempo Completo, Tiempo Parcial y Hora Cátedra) que involucran en su actividad académica las funciones de docencia, investigación, proyección social, gestión académica y desarrollo profesoral. Se encuentra constituida por estudiantes de nivel socioeconómico medio alto, que se agrupan en cinco Facultades (Ingeniería, Comunicación Social, Ciencias Económicas y Administrativas, Ciencias Básicas y Humanidades e Idiomas), los cuales son el centro del estudio, a través de las evaluaciones que realizan del desempeño de sus docentes.

El profesor Rizo (1999) señaló que en el contexto colombiano los elementos que más se utilizaban para interrogar a los estudiantes sobre el desempeño de sus docentes eran: cumplimiento, metodología, dominio y evaluación.

El Grupo de Investigación en Educación, ha estudiado durante varios años la problemática correspondiente a la evaluación académica y es conciente de la necesidad de generar espacios de reflexión en torno los elementos que constituyen factores clave de éxito en las políticas implementadas por las Instituciones de Educación Superior.

Siempre se ha mostrado un gran interés por investigar qué elementos están más allá de la calificación numérica que proporcionan los estudiantes periodo tras periodo sobre el desempeño de sus profesores.

El presente trabajo pretende continuar y consolidar el proceso de identificación de criterios e indicadores de evaluación que han sido fruto de la participación de todos los sectores académicos de la Institución, permitiendo que el docente y el estudiante se sientan parte activa de todo el proceso y asuman actitudes receptivas y propositivas hacia los resultados del mismo.

\section{MARCO REFERENCIAL}

En el momento de evaluar el desempeño de un docente se deben tener en cuenta todas las variables, sus interrelaciones y el entorno para emitir un juicio lo más objetivo posible 
Vásquez Rizo, F. E. y Gabalán Coello, J. (2006). Percepciones estudiantiles y su influencia en la evaluación del profesorado. Un caso en la Universidad Autónoma de Occidente, Cali-Colombia.

RELIEVE, v. 12, n. 2, p. 219-245. http://www.uv.es/RELIEVE/v12n2/RELIEVEv12n2 3.htm

de su actuación en el proceso de enseñanza aprendizaje y los actores que hacen parte de este proceso: profesores, estudiantes, institución, entorno social y sus interrelaciones.

"Es cada vez más evidente, en las Instituciones de Educación Superior, que la calidad educativa pasa por la calidad de la docencia que se imparte" (Rizo, 1999). El estudiante tiende a generalizar hacia la Universidad la opinión que tiene de sus profesores.

Es necesario asignar igual importancia a cada uno de los actores, ya que la retroalimentación entre ellos es multidireccional y permite establecer políticas y directrices encaminadas a la excelencia.

Rizo señala que "En este marco la evaluación docente debe romper los enfoques tradicionales que centrados en el control, enfatizan los procesos de medición y terminan por evaluar solo aquello que puede ser medido, descuidando aspectos que por el hecho de no poderse medir "objetivamente" no pueden dejar de evaluarse.".

"Emitir un juicio absoluto sobre la eficacia del profesor es prácticamente imposible, ya que éste no solo depende de él mismo (factores internos), sino también de los alumnos a los que dirige y de las condiciones y circunstancias en que se encuentre colocado (factores externos)", Juan Manuel Moreno (1972).

Hoy en día, la calidad docente se constituye en factor fundamental para determinar la competitividad de las Instituciones de Educación Superior, lo que demanda la creación de políticas que garanticen y aseguren la efectividad del proceso. Al respecto el profesor Daniel Meade (1996) comenta que en el sector educativo, a nivel general, "se ha logrado un avance considerable en la implantación de la calidad en el área académica a través de la interpretación en términos de procesos de enseñanza-aprendizaje y su medición utilizando sistemas de evaluación basados en indicadores de calidad".
Los estudios sobre la evaluación de la eficacia docente, teniendo en cuenta la percepción estudiantil, son muy variados; como indica Herbert Marsh (1982) “...estas puntuaciones son difícilmente validables porque no hay un criterio universal... si los indicadores de calidad y las puntuaciones de los estudiantes coinciden junto con alguna otra medida de la efectividad docente... entonces habrá fundamento para defender la validez.".

En relación al profesor y su grado de protagonismo en el proceso de evaluación docente, hay que tener en cuenta que no todos los profesores son pedagogos. Cristina Mayor Ruiz (1996) afirma al respecto: “...los profesores universitarios se limitan a imitar a sus profesores, y de esta forma, probablemente, han aprendido a enseñar, por ensayo y error".

En ese sentido, el profesor universitario debe ser un individuo íntegro que fusione sus conocimientos en el campo científico o técnico con su proceder como formador de personas útiles a la sociedad. Para Rizo (2004) "...se requiere con mayor urgencia que el profesor universitario, investigue, enseñe y se proyecte socialmente. Para ello, se requiere fundamentalmente de dos cosas: del desarrollo profesional de los docentes ligado a su contexto particular y de las mejora de las prácticas educativas".

En las Instituciones de Educación Superior, el estudiante es una fuente de información en el proceso de evaluación del rendimiento del profesor a lo largo de los periodos académicos. Los estudiantes son los destinatarios de un servicio educativo. Lo que sucede en el aula solo ellos lo pueden saber y expresar. No se pretende que juzguen a sus maestros sino que indiquen qué tipo de acciones se están dando en el aula y cómo están ocurriendo. Todo lo anterior justifica la importancia de las opiniones de los estudiantes en torno al proceso de evaluación del profesorado. Una observación que vale la pena anotar es que, teniendo en cuenta que por tratarse de 
Vásquez Rizo, F. E. y Gabalán Coello, J. (2006). Percepciones estudiantiles y su influencia en la evaluación del profesorado. Un caso en la Universidad Autónoma de Occidente, Cali-Colombia.

RELIEVE, v. 12, n. 2, p. 219-245. http://www.uv.es/RELIEVE/v12n2/RELIEVEv12n2 3.htm

percepciones se debe tener un manejo cuidadoso, debido a que obedecen a esquemas individuales y poseen cierto grado de subjetividad.

Con respeto a lo anterior, Rizo (2004) formula algunos interrogantes en torno a considerar al estudiante como fuente de información y cómo es un error utilizarlo como único analizador de la labor docente:

- “A A todos los estudiantes se les puede interrogar sobre todos los aspectos del trabajo de sus profesores?".

- ¿Valdría la pena presentar cuestionarios de opinión diferentes para estudiantes de primeros semestres que para los de semestres intermedios o para los de últimos semestres?".

- ¿Los estudiantes solo pueden dar información válida sobre los procesos de enseñanza aprendizaje?".

- ¿Es necesario cruzar información de rendimiento de los estudiantes con la información obtenida de sus percepciones acerca del trabajo del profesor?".

- ¿El rendimiento de los estudiantes es un buen indicador del trabajo del profesor?".

- ¿Si a los estudiantes les va bien es mérito del profesor y si les va mal se debe a un mal trabajo del profesor?".

Para José Antonio Arnaz y Teresa Yurén (1994) “...debe evitarse el medir la labor del docente en términos meramente cuantitativos, así como el establecer comparaciones con respecto a otros maestros; la evaluación debe orientarse a la obtención de un perfil del docente con respecto al modelo educativo".

En este sentido, se considera que el proceso de evaluación del desempeño docente debe definir el perfil del profesor, más acorde con un modelo educativo institucional, de tal manera que sirva como medio de mejoramiento de la práctica educativa.

La Institución es entonces, la encargada de propiciar un ambiente adecuado, donde estudiantes y profesores; que por constitución natural se encuentran cargados de valores subjetivos; se integren en la consolidación de un modelo de evaluación docente acorde con las necesidades de cada uno de los actores.

El entorno social, que determina el nivel de subjetividad de cada uno de los actores que participan en este proceso, se constituye en factor fundamental en el momento de emitir una apreciación, por parte del estudiante, en torno a su profesor.

“... la conducta de una persona es el resultado directo de su campo de percepciones en el momento de su comportamiento" (García y Villa, 1984).

El análisis de las interrelaciones entre profesores, estudiantes, institución y entorno social constituyen un punto de partida para determinar qué aspectos, según los estudiantes, son los más relevantes en el momento de emitir juicios evaluativos sobre el desempeño general de sus docentes, debido a que cada uno de estos actores afectan, de una $\mathrm{u}$ otra manera, la percepción.

Daniel Meade (1996) sostiene que "El profesor de calidad cambia de papel y se convierte en un facilitador que crea un ambiente de aprendizaje no solamente en el aula, sino también, y más aún, fuera del aula a través de actividades extracurriculares.".

En general, el modelo de enseñanza aprendizaje implementado en los procesos de modernización académica de las Instituciones de Educación Superior apunta hacia la formación, no solo de profesionales en áreas específicas del conocimiento, sino también de individuos con clara formación humanística y creativa. Esto implica que el docente se involucre con el estudiante más allá de actividades meramente curriculares y se vuelva permeable ante la percepción de sus alumnos.

Es importante que las relaciones entre los actores del proceso de evaluación docente se cohesionen de tal manera que constituyan un 
Vásquez Rizo, F. E. y Gabalán Coello, J. (2006). Percepciones estudiantiles y su influencia en la evaluación del profesorado. Un caso en la Universidad Autónoma de Occidente, Cali-Colombia.

RELIEVE, v. 12, n. 2, p. 219-245. http://www.uv.es/RELIEVE/v12n2/RELIEVEv12n2 3.htm

todo que gire en torno a un proceso articulado. (García y Villa, 1984) argumentan: “A veces el que termina siendo eficaz en la transformación del profesor es el alumno y el ambiente institucional.".

En estudios anteriores, el profesor Rizo (1999), encontró los siguientes resultados:

- La variable cumplimiento no afecta significativamente los resultados obtenidos por los docentes en las otras variables.

- La claridad con que un docente realiza sus explicaciones de clase es el indicador que más tienen en cuenta los estudiantes para calificar la labor de sus profesores.

- Cuando un docente realiza aplicaciones de su disciplina en otros campos del saber, consigue elevar la participación de los estudiantes en las clases.

- El cumplimiento de los horarios de clase por parte del profesor no afecta a la percepción que tiene el estudiante de su trabajo; en cambio, si el profesor incumple sus horarios resulta mal evaluado en la variable metodología.

- Hay una correlación significativa (más de 0.7 en el indicador de Pearson) entre los indicadores de dominio de la asignatura y la claridad con que se responden las preguntas de los estudiantes.

Además de las anteriores correlaciones, que son generalizadas, existen algunas correlaciones por sectores académicos que es importante resaltar:

- Para los cursos de Ciencias Económicas, los docentes que responden con claridad las preguntas que se les formulan, motivan a los estudiantes a profundizar en sus conocimientos.

- En los cursos de Ingeniería, los docentes que son claros en sus explicaciones permiten el desarrollo de habilidades y destrezas en sus estudiantes.

- En Comunicación Social hay una buena correlación entre el ítem correspondiente a dominio demostrado en la asignatura y la claridad con que se realizan las explicaciones por parte del docente.
- En los cursos de Ciencias Básicas, los profesores que fomentan la autoevaluación en sus estudiantes utilizan las evaluaciones como medio de aprendizaje y motiva a los estudiantes a profundizar en sus conocimientos.

De igual manera, en dicho estudio se concluye que los factores que menos influyen en el desempeño profesoral son.

- Si el docente es atento y facilita la comunicación con los estudiantes cuando se necesita aclarar dudas; la asistencia puntual del docente a las diferentes actividades programadas en el curso; si la bibliografía presentada por el profesor es coherente con los contenidos desarrollados en el curso y si los contenidos del curso se han desarrollado según los tiempos establecidos para ello.

Además, Rizo concluye que los factores que más influyen en el desempeño del profesor, según los estudiantes son:

- La claridad con que realiza las exposiciones, la claridad con que responde a las preguntas que se formulan y si las calificaciones que asigna el profesor son justas.

- En Comunicación Social influye, además de los anteriores, si el profesor motiva y fomenta la participación de los estudiantes.

- En Economía e Ingeniería, otro factor que influye es la entrega puntual dentro del periodo previsto de la entrega de las notas de las diferentes evaluaciones.

Finalmente, la evaluación docente debe ser entendida como un proceso en el cual se identifican fortalezas y oportunidades de mejoramiento, de tal manera que el docente pueda incrementar sus fortalezas y trabajar en sus debilidades obteniendo resultados satisfactorios para él, los estudiantes, el entorno social y la Institución en general.

Al respecto, Etxegaray y Sanz (1991) afirman que "... se debe hacer conscientes a los profesores de que la evaluación formativa de la labor docente es necesaria (a todos los 
Vásquez Rizo, F. E. y Gabalán Coello, J. (2006). Percepciones estudiantiles y su influencia en la evaluación del profesorado. Un caso en la Universidad Autónoma de Occidente, Cali-Colombia.

RELIEVE, v. 12, n. 2, p. 219-245. http://www.uv.es/RELIEVE/v12n2/RELIEVEv12n2 3.htm

niveles de enseñanza), si pretendemos avanzar en la mejora de la misma.".

\section{METODOLOGÍA DE TRABAJO UTILIZADA}

En la presente investigación se han utilizado procedimientos tanto cuantitativos como cualitativos, a partir de los cuales se intenta realizar una mirada holística al objeto de la investigación, integrando los dos paradigmas.

Los datos utilizados para el análisis cuantitativo se derivan de los resultados del cuestionario de opinión para alumnos: "Evaluación del Desempeño Docente", diseñado y aplicado semestralmente por el Centro de Desarrollo Académico de la Universidad Autónoma de Occidente. Desde esta perspectiva, al ser la Evaluación del Desempeño Docente un proceso institucional, el cuestionario es aplicado a todos los estudiantes, a partir de cada uno de los cursos ofrecidos por la Universidad, destinando 20 minutos de una clase regular, bajo supervisión de personal calificado de la Vicerrectoría Académica.

Cabe indicar, que el profesor no tiene ingerencia en este proceso debido a que en el momento de aplicar la evaluación se ausenta. De igual manera, el estudiante conserva el anonimato ya que el cuestionario no debe estar acompañado del nombre de quien lo diligencia.

Este cuestionario se constituye en una herramienta confiable que recoge de manera sistemática la opinión que tienen los estudiantes de sus profesores; en aspectos tales como: responsabilidad y compromiso institucional, metodología, dominio de la asignatura y evaluación.

El instrumento de recolección de información cuantitativa (cuestionario de opinión para alumnos) ha pasado por una depuración exhaustiva, como se muestra más adelante, de tal manera que el instrumento garantice la validez y la confiabilidad en torno a las opi- niones del estudiantado. En la misma dirección, la validez estadística de las preguntas formuladas está demostrada a partir de la afirmación que ninguna de las preguntas es explicada por otra, sustentado por estudios de multicolinealidad, donde se certifica la no existencia de colinealidad entre las variables, situación que axiomáticamente indica que la percepción que tienen los estudiantes sobre una pregunta específica no es explicada por la percepción observada en cualquier otra pregunta (en caso contrario, una de las dos podrá ser eliminada en tanto que las dos explican lo mismo). De igual manera, cabe anotar que se ha realizado una reflexión al interior de la Institución sobre la pertinencia de las variables involucradas en el cuestionario y sobre la necesidad de conocer la percepción de los estudiantes en cada uno de los tópicos que conforman dicho cuestionario.

En términos cualitativos, en torno a la pregunta No. 15 (variable respuesta), se realizan entrevistas grupales, en las cuales se recogen opiniones a nivel informal, analizando desde otra perspectiva las impresiones de algunos estudiantes.

Se escoge la pregunta No. 15 del cuestionario "En general y de acuerdo a su desempeño, indique la calificación que le asigna al profesor", debido a que ésta recoge la impresión universal que los estudiantes poseen de sus profesores y a la vez juega un papel estadístico importante porque actúa como eje central de la interacción de las 14 preguntas restantes. Esta pregunta (15) fue incorporada a partir del año 2000 en el cuestionario gracias a un riguroso proceso de análisis, el cual concluyó que era necesario tener un punto de referencia que sintetizara la opinión del estudiantado y que permitiera interrelacionar las variables de medición.

Se trabaja en dos grandes campos, para facilitar el análisis y la toma de decisiones en cuanto a la ruta que debe seguir el trabajo investigativo, tanto en su parte cuantitativa como en su parte cualitativa. 
Vásquez Rizo, F. E. y Gabalán Coello, J. (2006). Percepciones estudiantiles y su influencia en la evaluación del profesorado. Un caso en la Universidad Autónoma de Occidente, Cali-Colombia.

RELIEVE, v. 12, n. 2, p. 219-245. http://www.uv.es/RELIEVE/v12n2/RELIEVEv12n2 3.htm

El primero de esos campos está integrado por aquellas asignaturas exclusivas de cada Programa de Formación Académica, entendiéndose por exclusivas aquellas asignaturas que hacen parte del área de formación profesional, la cual contiene a su vez dos componentes: a) Formación básica profesional: orientado a suministrar una sólida formación al estudiante en aquello que constituye los conocimientos habilidades destrezas y competencias básicas de su profesión y b) Componente de énfasis: conjunto de cursos orientados a iniciar al estudiante en un área específica del conocimiento, dentro o fuera de su profesión, de tal forma que adquiera competencia mínima para desempeñarse en ese campo específico (Universidad Autónoma de Occidente, 2002).

El segundo campo se encuentra constituido por asignaturas comunes, que hacen parte de los primeros tres semestres de todos los Programas de Formación Académica de la Institución, entendiéndose asignaturas comunes aquellas que hacen parte del área de formación básica, la cual contiene tres componentes: a) Componente de fundamentación: orientado a fortalecer los conocimientos de los nuevos estudiantes en aquellas temáticas fundamentales para la comprensión de problemas en las profesiones específicas; b) Componente de formación humanística: orientado a formar al estudiante con una visión clara de su integralidad como ser humano en todas sus dimensiones (espiritual, cognitiva, afectiva, física, ética, estética, social, política, comunicativa y ecológica) y c) Componente investigativo: identificado como el proceso pedagógico mediante le cual se pretende familiarizar al estudiante con este tipo de procedimientos, en cuanto a visiones lógicas, dinámicas, métodos, problemáticas y actividades propias de la investigación (Universidad Autónoma de Occidente, 2002).

Se opta por esta distribución debido a que se considera, a partir de conclusiones de estudios anteriores, que los estudiantes que hacen parte del primer campo tienen más elementos de juicio en el momento de calificar el desempeño de sus profesores. En el caso de los estudiantes del segundo campo, éstos constituyen un factor igualmente importante debido a que su percepción, si bien no tiene elementos de juicio tan claros como los anteriores, no se encuentra influenciada por la cultura universitaria de la evaluación del desempeño docente, lo que permite auscultar una posición "natural".

\section{- Para el análisis cuantitativo $^{[1]}$}

La encuesta se define como una técnica destinada a obtener información primaria a partir de un número representativo de individuos de una población, para proyectar sus resultados sobre la población total.

En el presente artículo se aborda este instrumento por medio del cuestionario "Evaluación del Desempeño Docente".

Los elementos constitutivos de una encuesta son tenidos en cuenta en la fase de diseño del cuestionario. Estos elementos son: definición y operacionalización de los conceptos de la encuesta, diseño del cuestionario (construir las preguntas y disponerlas ordenadamente), diseño de la muestra, trabajo de campo y registro de la encuesta.

Esta información determina los procedimientos que se realizan desde una perspectiva cuantitativa, utilizando algunos métodos multivariantes.

En esta investigación se utiliza un análisis de correspondencias, que permite identificar tendencias en torno a la pregunta No. 15.

Para poder identificar el grado de relevancia de las 14 preguntas del cuestionario con la calificación que indaga sobre el desempeño general de los profesores se recurre a utilizar un método de estadística multivariada denominado análisis discriminante, el cual permite determinar el grado de relevancia de las variables del cuestionario, sobre la calificación general que se hace del docente, y 
Vásquez Rizo, F. E. y Gabalán Coello, J. (2006). Percepciones estudiantiles y su influencia en la evaluación del profesorado. Un caso en la Universidad Autónoma de Occidente, Cali-Colombia.

RELIEVE, v. 12, n. 2, p. 219-245. http://www.uv.es/RELIEVE/v12n2/RELIEVEv12n2 3.htm

cuáles de ellas tienen un mayor peso o ponderación en la percepción final.

En el Anexo 1 se presentan los tópicos abordados por el cuestionario "Evaluación del Desempeño Docente" que se utiliza en la UAO.

\section{- Para el análisis cualitativo}

Para llevar a cabo una interpretación adecuada del análisis de correspondencias y contrastar los factores externos que influyen en la percepción de la pregunta No. 15 en el análisis discriminante, que se enuncia más adelante, se involucra la visión cualitativa a través de entrevistas grupales, empleando dos métodos fundamentales de muestreo, descritos por Michael Quin Patton (1987):

1.Muestreo con Base en Criterios: Revisa y estudia todos los casos que poseen algunos criterios de importancia predeterminados.

Se selecciona este método debido a que el punto clave de esta modalidad de muestreo es tener la seguridad de entender los casos que potencialmente pueden ser ricos en información, dado que pueden revelar las posibles debilidades del sistema, y que pueden ser blanco de oportunidad para el programa y la mejora del sistema.

2.Muestreo con base en Casos Confirmatorios y Disconfirmatorios: Es utilizado con el fin de probar ideas, confirmar la importancia y significado de los patrones identificados y revisar la viabilidad de los hallazgos.

Este método es empleado porque el componente cuantitativo del proyecto arroja algunas tendencias que deben ser contrastadas, explicadas, ampliadas, refutadas o reafirmadas por los integrantes del focus group.

La entrevista grupal se realiza con base en las percepciones abiertas de los estudiantes, quienes contestan preguntas establecidas por los investigadores, basadas en observaciones realizadas y tomando en cuenta el comportamiento de las respuestas del cuestionario.
Se reúnen grupos de estudiantes (entre seis $\mathrm{y}$ ocho personas) por Facultad, en un ambiente amigable y se entabla una conversación que permite conocer de manera informal sus opiniones, buscando que sean grupos caracterizados por su importante nivel de compromiso institucional.

El instrumento a nivel procedimental exige elaborar un cuestionario que abarque los mismos tópicos de la encuesta de evaluación, con el fin de confirmar tendencias y aspectos externos, de tal manera que se puedan identificar aspectos ajenos, tenidos en cuenta por ellos, que no mide el cuestionario.

\section{DESARROLLO DE LA INVESTI- GACIÓN}

\subsection{Consideraciones}

La pregunta No. 15 se concibe como la pregunta que agrupa la opinión contenida en las anteriores, por lo tanto debe existir una asociación entre las calificaciones asignadas en las 14 preguntas iniciales y la opinión final, reflejada en la pregunta No. 15.

Según la clasificación de las correlaciones elaboradas por Colton ${ }^{[2]}$, se puede decir que existe una asociación moderada - buena entre cada una de las preguntas con la pregunta imagen (15). Lo que permite inferir su calidad de variable dependiente.

\subsection{Análisis de correspondencias en el marco del proceso de evaluación}

Se desarrolla un análisis de correspondencias para identificar percepciones estudiantiles comunes o tendencias intraprogramas e interprogramas en torno a la calificación otorgada en el desempeño general de los profesores de la Universidad Autónoma (pregunta No. 15 en el cuestionario). Una vez realizado este procedimiento se recurre a la interpretación complementaria del mismo a partir de los resultados arrojados por las entrevistas grupales. 
Vásquez Rizo, F. E. y Gabalán Coello, J. (2006). Percepciones estudiantiles y su influencia en la evaluación del profesorado. Un caso en la Universidad Autónoma de Occidente, Cali-Colombia.

RELIEVE, v. 12, n. 2, p. 219-245. http://www.uv.es/RELIEVE/v12n2/RELIEVEv12n2 3.htm

La importancia del análisis de correspondencias, método de análisis de datos multivariados, radica en que por un lado brinda la posibilidad de identificar tendencias alrededor de las percepciones estudiantiles sobre los ítems tenidos en cuenta en el momento de calificar el desempeño de sus docentes; y por otro permite abordar la semejanza en las percepciones de estudiantes de diferentes carreras.

\section{- ¿En qué consiste un análisis de co- rrespondencias?.}

"Es una técnica descriptiva para representar tablas de contingencia, es decir, tablas donde recogemos las frecuencias de aparición de dos o más variables cualitativas en un conjunto de elementos". "La información de partida es una matriz de dimensiones I x $\mathrm{J}$ que representa las frecuencias absolutas observadas de dos variables cualitativas en $n$ elementos. La primera variable se representa por filas, y suponemos que toma I valores posibles y la segunda se representa por columnas, y toma $\mathrm{J}$ valores posibles" (Peña, 2002).

"En lugar de comparar filas / columnas, el análisis de correspondencias procede derivando un pequeño número de dimensiones, de tal forma que la primera dimensión (primer eje o factor) "explique" la mayor parte de la asociación total entre filas y columnas (asociación medida en términos de $\mathrm{X}^{2} / \mathrm{N}$ ), la segunda dimensión (segundo eje o factor) explique la mayor parte de la asociación no explicada por la primera (residuo) y así sucesivamente con el resto de dimensiones. El máximo número de dimensiones es igual al menor número de modalidades de cualquiera de las variables (fila o columna) menos uno, pero generalmente, dos o tres dimensiones son suficientes para representar adecuadamente la asociación entre ambas variables. La cantidad $\mathrm{X}^{2} / \mathrm{N}$ se denomina inercia total. Esta inercia se descompone en un total de $\mathrm{k}$

Este procedimiento requiere además, cuando se realiza el Gráfico No. 1, interpretar valores característicos (propios) cada uno de los cuales constituye la inercia principal de una dimensión" (Díaz, 2002).

\section{- Pruebas de dependencia a partir del estadístico chi-cuadrado.}

Se intenta con esta prueba determinar si existe algún grado de dependencia entre la percepción de los estudiantes y el Programa al cual pertenecen, es decir identificar si los Programas imprimen algún sello distintivo en cuanto a las maneras de pensar y concebir el proceso evaluativo por parte de los estudiantes.

Para la pregunta No. 15 "En general y de acuerdo con su desempeño, indique la calificación que le asigna al profesor", la Tabla No. 1 permite observar que la proporción total de inercia explicada, por las dos dimensiones es de $91.7 \%$, lo que permite concluir que existe un alto nivel de asociación entre los Programas y las calificaciones otorgadas.

La significancia del estadístico Chicuadrado es alta, lo que quiere decir que existe dependencia entre la calificación general que los estudiantes asignan a sus profesores y el Programa de Formación Académica al cual pertenecen; en otros términos, se evidencia que la apreciación o percepción de los estudiantes con respecto a sus docentes está condicionada por el Programa de origen.

\section{TABLA No. 1}



cada una de las dimensiones generadas (ejes coordenados) de acuerdo al nivel de aporte de cada una de las variables a éstas. Dicha 
Vásquez Rizo, F. E. y Gabalán Coello, J. (2006). Percepciones estudiantiles y su influencia en la evaluación del profesorado. Un caso en la Universidad Autónoma de Occidente, Cali-Colombia.

RELIEVE, v. 12, n. 2, p. 219-245. http://www.uv.es/RELIEVE/v12n2/RELIEVEv12n2 3.htm

ponderación no arroja resultados estructurales, luego se utiliza una interpretación de acuerdo a la cercanía de las dos variables contrastadas (calificación general del docente y Programa de Formación Académica).

\section{- Gráficos de puntos de columna y de} fila y confrontación a partir de los grupos focales.

El Gráfico No. 1 ilustra la manera en que se interrelacionan las diferentes calificaciones otorgadas y cada uno de los Programas de Formación. En esta dirección, la cercanía de un Programa a una calificación indica la tendencia a calificar a sus profesores, en cualquier valor de la escala de medición ordinal.

\section{GRÁFICO No.1}

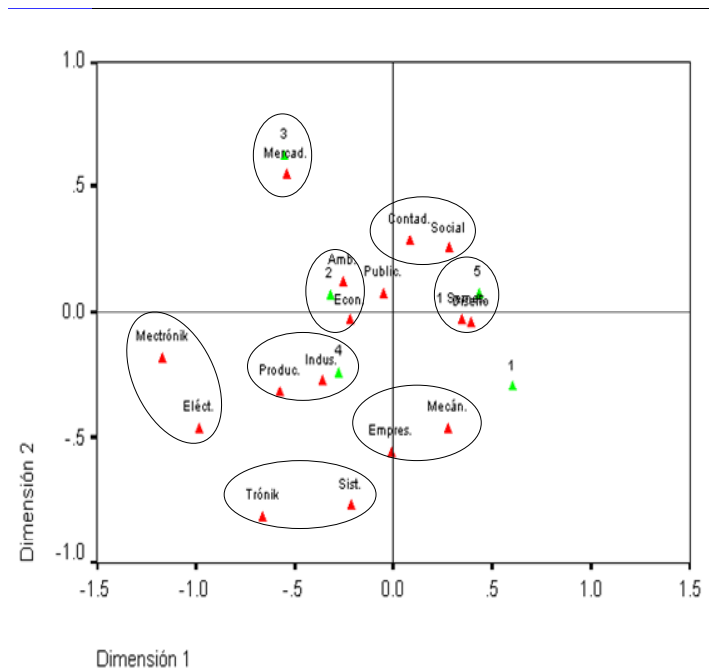

Se observa en el Gráfico No. 1, y encerradas en óvalos, las asociaciones que existen entre las calificaciones y los Programas o entre percepciones similares entre Programas. Estas tendencias, en la investigación, se tratan de explicar teniendo en cuenta las opiniones evidenciadas en las entrevistas grupales.

Sin embargo, se debe considerar que por tratarse de percepciones, que aportan elementos valiosos al proceso, no deben ser la única fuente de evaluación del desempeño del profesor, debido al grado de subjetividad que tienen intrínseco.

Los estudiantes del Programa de Mercadeo tienen dependencia con la calificación 3 , lo que permite identificar cierta tendencia a la calificación de sus profesores de manera regular. Al respecto, los estudiantes en su participación en las entrevistas grupales, manifiestan su inconformidad a partir de los siguientes comentarios: "He criticado mucho la calidad de los docentes porque hay unos profesores que no tienen idea de la calidad docente. Aunque hay otros muy buenos". "Creo que a algunos les falta experiencia para ser excelentes". "Algunos profesores llegan al salón y a uno no le dan ni ganas de escucharlo". "A veces uno piensa -lo que me está diciendo este profesor lo puedo conseguir en Internet y lo entiendo mejor-. En ese sentido falta mucha calidad en los profesores". "Hay unos que si son muy buenos pero hay otros que realmente están desenfocados".

Existe tendencia hacia la calificación de excelencia (5) a partir de las percepciones de los estudiantes adscritos al Programa de Diseño de la Comunicación Gráfica y los estudiantes de Primeros Semestres. En los focus group se establecen los siguientes elementos: "La calidad de mis profesores ha sido buena, por ahora todos los profesores que me han tocado han sido de calidad". "La calidad de los profesores es buena y uno aprende. Cuando me va mal en una materia depende de mí, porque no me dedico, pero a uno si le aclaran al comienzo que tiene que dedicar al estudio, por cada hora de clase dos por fuera, pero uno no hace eso". De manera particular, los estudiantes de Primeros Semestres manifiestan que la calificación en torno al 5 se puede otorgar bajo dos situaciones: 1. Desde el punto de vista de la excelente calidad que ostentan los profesores de los Primeros Semestres de la Universidad y 2. Se fundamenta en la poca experiencia que tienen en el proceso de evaluación y el escaso número de 
Vásquez Rizo, F. E. y Gabalán Coello, J. (2006). Percepciones estudiantiles y su influencia en la evaluación del profesorado. Un caso en la Universidad Autónoma de Occidente, Cali-Colombia.

RELIEVE, v. 12, n. 2, p. 219-245. http://www.uv.es/RELIEVE/v12n2/RELIEVEv12n2 3.htm

profesores que han tenido, lo que impide que puedan realizar comparaciones.

También se infiere cierta similitud entre las apreciaciones de estudiantes de los Programas de Administración del Medio Ambiente y de los Recursos Naturales y de Economía, cuando se emiten juicios valorativos de sus docentes en torno a la calificación 2. Los estudiantes del Programa de Administración del Medio Ambiente y de los Recursos Naturales afirman: "Los docentes deben ser personas bien formadas y estructuradas, deben saber y manejar muy bien el tema que están enseñando y deben ser de gran calidad educativa". "La calidad en educación implica coherencia entre la metodología de enseñaza que se puede dar en un determinado Programa y los valores, así como una continua cualificación de los profesionales que conforman la Institución". "Los docentes deberían estar comprometidos con el Programa". "Si uno como estudiante no encuentra apoyo pues va a considerar malos a los profesores". "Lo que sucede es que nuestro Programa necesita profesores con un perfil más amplio, en cuanto a docencia, necesita personas que apoyen diferentes tipos de propuestas con la suficiente capacidad e idoneidad para orientar un proyecto y que tengan suficiente compromiso para dedicarse al estudiante". Los estudiantes del Programa de Economía afirman sobre la calidad de sus docentes: "Creo que algunos profesores no deberían estar dictando cátedra en esta Universidad porque no manejan bien el tema y no dan libertad a los estudiantes, nunca promueven un diálogo, un debate". "Hay profesores que creen que manejan la verdad absoluta y no saben que en las ciencias sociales (economía) la verdad absoluta no existe. Varios puntos de vista pueden tener la razón así sean opuestos lo que importa verdaderamente es la forma en que argumentamos ese tipo de conocimiento".

Percepciones similares, pero sin asociación a un puntaje particular, se presentan entre los estudiantes de los Programas de Ingeniería
Mecatrónica e Ingeniería Eléctrica, entre los estudiantes de Ingeniería Electrónica e Ingeniería Informática y de Sistemas, entre los estudiantes de Administración de Empresas e Ingeniería Mecánica y entre los estudiantes de Contaduría y Comunicación Social - Periodismo.

Para los estudiantes de los Programas de Ingeniería Mecatrónica e Ingeniería Eléctrica la calidad docente hace referencia a: "El problema es que los profesores tienden a volverse algo académicos y la experiencia se queda en un segundo plano". "Son profesores demasiado académicos. Algunos profesores cátedra miran más las aplicaciones a nivel industrial y se actualizan más". "Muchas veces ellos dan grandes definiciones, pero en el momento de enfrentarse a la realidad quedan en pañales y eso lo transmiten al estudiante". "Hay profesores muy buenos, que saben mucho, que tienen excelente pedagogía pero les falta experiencia externa de la industria que es la que realmente sirve". "Hay que definir primero que en nuestra carrera hay un ciclo básico en el que no le exijo mucho al profesor en experiencia, pero si hay un ciclo superior en el que sí necesitamos que el profesor tenga experiencia en la industria". "Que transmitan al estudiante esa experiencia, que sean el medio de comunicación entre la empresa y el mundo académico".

Las percepciones de los estudiantes de los Programas de Ingeniería Electrónica e Ingeniería Informática y de Sistemas se establecen de la siguiente manera: "Conozco profesores de planta muy preparados, que saben demasiado, que saben de pedagogía, pero muchas veces solamente han trabajado en la Universidad y no tienen la experiencia que se necesita". "Solo se quedan con lo que está en los libros". "Hay unos muy buenos que se preocupan por el estudiante, porque aprendamos, porque entendamos, pero hay otros que solo se limitan a dictar clase y algunos que ni siquiera van". "Nos ha tocado de todo". 
Vásquez Rizo, F. E. y Gabalán Coello, J. (2006). Percepciones estudiantiles y su influencia en la evaluación del profesorado. Un caso en la Universidad Autónoma de Occidente, Cali-Colombia.

RELIEVE, v. 12, n. 2, p. 219-245. http://www.uv.es/RELIEVE/v12n2/RELIEVEv12n2 3.htm

Los estudiantes de los Programas de Administración de Empresas e Ingeniería Mecánica manifiestan, en el momento de hablar sobre la calidad de sus profesores: "La calidad de los docentes ha mejorado muchísimo. Se ha visto que de algo han servido las evaluaciones que hacen y se ha visto que se han tenido en cuenta los comentarios que hemos puesto". "El cambio se ha visto y eso hay que rescatarlo". "Nosotros como estudiantes siempre queremos cambios. Yo estoy en noveno y los docentes que tengo son muy respetables, son muy buenos y así los he calificado, de pronto no pasaba esto con los anteriores". "Algunos profesores son de calidad, pero no todos". "He tenido profesores de todo tipo, de todas las edades y he encontrado que la metodología de los profesores antiguos no es tan práctica como la de los profesores nuevos". "El profesor nuevo sabe llegarle un poco más fácil al estudiante, el profesor joven es mucho más amable, es menos monótono". "No todos los profesores antiguos tiene mala metodología, también hay algunos que trabajan muy bien con el estudiante".

Las percepciones de los estudiantes de los Programas de Contaduría Pública y Comunicación Social - Periodismo son similares y se resumen a continuación: "En toda la carrera tratamos con muchos profesores, se tiene muy buena relación personal con el profesor pero igual en medio de esa relación personal le responde al profesor y sus trabajos son buenos y en medio de la amistad existe colaboración. Entonces uno lo califica bien".

La calificación deficiente (1) no se asocia con ningún Programa en particular, lo que constituye un punto importante para la UAO en cuanto a calidad docente, debido a que no existe una clara tendencia que permita vincular la calificación 1 con alguno de los Programas.

Por otra parte, los estudiantes pertenecientes a los Programas de Ingeniería de Producción e Ingeniería Industrial, demuestran cier- ta similitud en las percepciones alrededor del 4. En este sentido los estudiantes opinan: "Pienso que la calidad docente es algo integral, no es solamente que el profesor tenga una excelente pedagogía sino también que tenga preparación. He conocido durante toda la carrera profesores que tienen buena metodología y muchos estudios". "No es solamente el hecho que tenga la capacidad de transmitir conocimiento sino que tenga la suficiente capacitación y sobre todo que nos motive a la investigación". "Los profesores de la Universidad son de calidad". "Yo diría que en el Programa de Ingeniería Industrial hemos tenido el $70 \%$ de profesores de calidad. El 30\% restante no son de calidad".

4.3. Grado de relevancia de cada una de las preguntas en la opinión general del docente.

\section{- Consideraciones a nivel institucional}

Los objetivos principales abordados por el análisis discriminante son, por una parte la separación o discriminación de grupos, y por otra, la predicción o asignación de un objeto en un grupo entre varios previamente definidos, con base en los valores de las variables que lo identifican.

A nivel institucional el análisis discriminante permite establecer diferencias y clasificar los profesores, que según la percepción de sus estudiantes se encuentran divididos por el umbral de calidad definido por la Universidad Autónoma de Occidente (2002).

Para dicho análisis se lleva a cabo una recategorización de las calificaciones asignadas por los estudiantes en la pregunta No. 15, debido a que esta pregunta constituye la variable dependiente en el modelo contemplado. Las 14 preguntas anteriores interactúan de tal manera que afectan la percepción de los estudiantes en la pregunta pivote (15).

La pregunta No. 15, para efectos del presente trabajo investigativo, se divide en dos grupos: el primero está conformado por las 
Vásquez Rizo, F. E. y Gabalán Coello, J. (2006). Percepciones estudiantiles y su influencia en la evaluación del profesorado. Un caso en la Universidad Autónoma de Occidente, Cali-Colombia.

RELIEVE, v. 12, n. 2, p. 219-245. http://www.uv.es/RELIEVE/v12n2/RELIEVEv12n2 3.htm

opiniones de los estudiantes que califican a sus profesores por debajo de 3.6. El segundo grupo está constituido por aquellas percepciones que son iguales o superiores a este valor crítico. Como la escala de medición de la pregunta No. 15 es categórica ordinal, solo puede tomar los valores de 1, 2, 3, 4 y 5, se establece que para el primer grupo se consi- deran las percepciones que en la pregunta No. 15 oscilan entre 1 y 3 , y para el segundo grupo aquellas que lo hacen entre 4 y 5 .

Todo el proceso anterior es un insumo para la creación de un modelo discriminante con las siguientes características:

$$
\begin{gathered}
D=\beta_{1}(\mathbf{X} 1)+\beta_{2}(X 2)+\beta_{3}(X 3)+\beta_{4}(X 4)+\beta_{5}(X 5)+\beta_{6}(X 6)+\beta_{7}(X 7)+\beta_{8}(X 8)+\beta_{9} \\
(X 9)+\beta_{10}(X 10)+\beta_{11}(X 11)+\beta_{12}(X 12)+\beta_{13}(X 13)+\beta_{14}(X 14)+C
\end{gathered}
$$

Donde:

- D es la Variable Respuesta, que permite la clasificación, dependiendo de su valor, en los dos grupos mencionados.

- $\beta_{\mathrm{i}}$ es el coeficiente asociado a cada pregunta.

- $\mathrm{X}_{\mathrm{i}}$ representa el valor que toma cada pregunta en cada percepción.

"Frecuentemente la mejor función para separar grupos provee también la mejor regla de localización de observaciones futuras; de tal manera que estos dos términos generalmente se emplean indistintamente" (Díaz, 2002).

En el análisis discriminante se obtiene una función que permite separar varios grupos definidos a priori. Esta función es una combinación lineal de las variables de identificación, la cual minimiza los errores de clasificación. El problema de la discriminación es entonces comprobar si tales variables permiten diferenciar las clases definidas previamente y precisar cómo se puede hacer, utilizando para tal fin los datos obtenidos a través del cuestionario.

A partir del postulado anterior, lo que se intenta realizar es determinar que tan cercano o distante se encuentra la clasificación que se hace por medio de la modelización matemática de la opinión general que se tiene del docente contrastándolo con la calificación real que los estudiantes atribuyen a este ítem.

En cuanto a la correlación canónica ${ }^{[3]}$ evidenciada entre las percepciones sobre las características del docente y su desempeño a nivel general, se obtiene que el $47,47 \%$ de la variación en el juicio valorativo general que se hace del docente (P15) es explicada por la variación de cada una de las preguntas entre la 1 y la 14. Tabla No. 2.

\section{TABLA No. 2}

\begin{tabular}{|c|c|c|c|c|}
\hline Funcić & Autovalon & de varianz & zo acumulad & $\begin{array}{l}\text { Correlación } \\
\text { canónica }\end{array}$ \\
\hline 1 & $.903^{\mathrm{a}}$ & 100.0 & 100.0 & .689 \\
\hline
\end{tabular}

Autovalores

En términos conceptuales, esto quiere decir que existen otros factores, además de los mencionados por la encuesta, en los cuales los estudiantes se basan en el momento de evaluar el desempeño de sus docentes. Aproximadamente el 52,53\% de la variación en la variable de respuesta (evaluación general del docente) es explicada por fenómenos externos al cuestionario. 
Vásquez Rizo, F. E. y Gabalán Coello, J. (2006). Percepciones estudiantiles y su influencia en la evaluación del profesorado. Un caso en la Universidad Autónoma de Occidente, Cali-Colombia.

RELIEVE, v. 12, n. 2, p. 219-245. http://www.uv.es/RELIEVE/v12n2/RELIEVEv12n2 3.htm

Como se ha visto, influyen otros aspectos como el nivel de empatía que desarrolle el profesor con los estudiantes, reflejado en relaciones amistosas, aspecto personal y humano del docente, número de docentes que se hayan tenido a lo largo de la carrera (a través del estudio se ha observado que los estudiantes de primeros semestres califican de una mejor manera a sus profesores) y demás aspectos que se han dejado ver por medio de la metodología de entrevistas grupales.

Esta consideración, corrobora una vez más la necesidad de un análisis cualitativo a través de entrevistas grupales, explicadas en párrafos anteriores, para conocer las percepciones de los estudiantes en torno a la temática tratada y determinar qué otros factores inciden en la variación de la opinión con respecto al desempeño general del docente.

De igual manera, el estudio permite determinar que sí se presentan diferencias significativas entre los centroides de cada una de las preguntas, cuando se realiza la partición en los dos grupos generados por el carácter dicotómico de la variable respuesta. Esto determina que al existir diferencias la clasificación y el proceso de discriminación es más efectivo porque la distancia entre los medias es grande y permite una mayor diferenciación de los grupos estudiados. En esta dirección, existirá una mayor diferenciación entre los profesores que se encuentran por arriba del estándar definido por la Universidad con los profesores que se localizan por debajo del estándar de calidad, definido en las políticas de la Institución, en la medida en que se rechace la prueba de igualdad de medias. Esta observación se resume en el siguiente párrafo, debido a que los grupos se alejarán más y la discriminación será mayor.

La Tabla No. 3 realiza una prueba de igualdad de medias de los grupos, en la que la hipótesis nula considera que las medias entre los grupos son iguales y la hipótesis alterna que la media entre los grupos no lo es. Es necesario que se rechace la hipótesis nula porque en la medida que sean diferentes dichas medias o centroides se podrán clasificar las percepciones de los estudiantes con menor grado de error en dicha estimación. En el caso particular se rechaza la hipótesis nula para todas las preguntas de una manera altamente significativa, indicando que cada una de éstas sirve para realizar una discriminación adecuada.

$$
\begin{aligned}
& \text { Ho: } \mu_{1}=\mu_{2} \\
& \text { Ha: } \mu_{1} \neq \mu_{2}
\end{aligned}
$$


Vásquez Rizo, F. E. y Gabalán Coello, J. (2006). Percepciones estudiantiles y su influencia en la evaluación del profesorado. Un caso en la Universidad Autónoma de Occidente, Cali-Colombia.

RELIEVE, v. 12, n. 2, p. 219-245. http://www.uv.es/RELIEVE/v12n2/RELIEVEv12n2 3.htm

TABLA No. 3

Pruebas de igualdad de las medias de los grupos

\begin{tabular}{|c|c|c|c|c|c|}
\hline & $\begin{array}{l}\text { Lambda } \\
\text { de Wilks }\end{array}$ & $\mathrm{F}$ & gl1 & $g \mid 2$ & Sig. \\
\hline $\begin{array}{l}\text { El profesor cumple responsablemente con el desarrollo del } \\
\text { curso }\end{array}$ & .767 & 2901.200 & 1 & 9528 & .000 \\
\hline $\begin{array}{l}\text { El profesor contribuye al cumplimiento de la misión institucional } \\
\text { fomentando en sus estudiantes una visión humanística, } \\
\text { creativa y emprendedora }\end{array}$ & .723 & 3647.332 & 1 & 9528 & .000 \\
\hline $\begin{array}{l}\text { El profesor contribuye a la generación de conocimiento y a la } \\
\text { solución de los problemas del entorno regional, nacional e } \\
\text { internacional }\end{array}$ & .753 & 3126.647 & 1 & 9528 & .000 \\
\hline El profesor facilita el aprendizaje de los estudiantes & .684 & 4409.150 & 1 & 9528 & .000 \\
\hline El docente propicia el auto - aprendizaje de los estudiantes & .748 & 3210.670 & 1 & 9528 & .000 \\
\hline El profesor promueve la participación del estudiante & .737 & 3393.632 & 1 & 9528 & .000 \\
\hline $\begin{array}{l}\text { El profesor conserva en su comunicación con el estudiante una } \\
\text { relación de mutuo respeto }\end{array}$ & .771 & 2834.001 & 1 & 9528 & .000 \\
\hline $\begin{array}{l}\text { El profesor utiliza las evaluaciones como medio para mejorar el } \\
\text { aprendizaje }\end{array}$ & .720 & 3701.566 & 1 & 9528 & .000 \\
\hline $\begin{array}{l}\text { El profesor manifiesta en su discurso y en la manera de llevar } \\
\text { el curso, dominio sobre el campo de conocimiento de su } \\
\text { asignatura }\end{array}$ & .732 & 3494.590 & 1 & 9528 & .000 \\
\hline $\begin{array}{l}\text { El profesor brinda orientaciones y alternativas claras y precisas } \\
\text { a las preguntas que se le formulan }\end{array}$ & .703 & 4030.648 & 1 & 9528 & .000 \\
\hline La propuesta de evaluación establecida es precisa y coherente & .722 & 3676.707 & 1 & 9528 & .000 \\
\hline $\begin{array}{l}\text { El profesor orienta y fomenta la autoevaluación por parte de los } \\
\text { estudiantes }\end{array}$ & .720 & 3714.510 & 1 & 9528 & .000 \\
\hline $\begin{array}{l}\text { El profesor además de los parciales realizados tiene en cuenta } \\
\text { otras actividades realizadas por los estudiantes para la } \\
\text { evaluación del curso }\end{array}$ & .748 & 3218.013 & 1 & 9528 & .000 \\
\hline Las calificaciones asignadas por el profesor han sido justas & .694 & 4195.224 & 1 & 9528 & .000 \\
\hline
\end{tabular}

Las preguntas que discriminan mejor, a partir del estadístico F, son las siguientes:

- "El profesor facilita el aprendizaje de los estudiantes" ( $\mathrm{F}=4409.50)$.

- "Las calificaciones asignadas por el profesor han sido justas" $(\mathrm{F}=4195.224)$.

- "El profesor brinda orientaciones y alternativas claras y precisas a las preguntas que se le formulan" ( $F=4030.648)$.

- "El profesor orienta y fomenta la autoevaluación por parte de los estudiantes" (F $=3714.510$ ).

- "El profesor utiliza las evaluaciones como medio para mejorar el aprendizaje" (F $=3701.566$ ).

La Tabla No. 3 se relaciona con las Tablas Nos. 4 y 5. En la Tabla No. 4 se presenta la correlación de las variables (preguntas) con la función discriminante (modelo) y en la
Tabla No. 5 se analizan los coeficientes que acompañarán a cada pregunta.

La Tabla No. 4 presenta la matriz de estructura, la cual permite concluir que las variables (preguntas) que tienen mayor correlación con el modelo o función discriminante son las siguientes:

- "El profesor facilita el aprendizaje de los estudiantes" $(\mathrm{Co}=0.716)$.

- "Las calificaciones asignadas por el profesor han sido justas" $(\mathrm{Co}=0.698)$.

- "El profesor brinda orientaciones y alternativas claras y precisas a las preguntas que se le formulan" $(\mathrm{Co}=0.685)$.

- "El profesor orienta y fomenta la autoevaluación por parte de los estudiantes" $(\mathrm{Co}=0.657)$.

- "El profesor utiliza las evaluaciones como medio para mejorar el aprendizaje" $(\mathrm{Co}=0.656)$. 
Vásquez Rizo, F. E. y Gabalán Coello, J. (2006). Percepciones estudiantiles y su influencia en la evaluación del profesorado. Un caso en la Universidad Autónoma de Occidente, Cali-Colombia.

RELIEVE, v. 12, n. 2, p. 219-245. http://www.uv.es/RELIEVE/v12n2/RELIEVEv12n2 3.htm

TABLA No. 4

Matriz de estructura

\begin{tabular}{|c|c|}
\hline & Función \\
\hline & 1 \\
\hline El profesor facilita el aprendizaje de los estudiantes & .716 \\
\hline Las calificaciones asignadas por el profesor han sido justas & .698 \\
\hline $\begin{array}{l}\text { El profesor brinda orientaciones y alternativas claras y precisas a las } \\
\text { preguntas que se le formulan }\end{array}$ & .685 \\
\hline $\begin{array}{l}\text { El profesor orienta y fomenta la autoevaluación por parte de los } \\
\text { estudiantes }\end{array}$ & .657 \\
\hline $\begin{array}{l}\text { El profesor utiliza las evaluaciones como medio para mejorar el } \\
\text { aprendizaje }\end{array}$ & .656 \\
\hline La propuesta de evaluación establecida es precisa y coherente & .654 \\
\hline $\begin{array}{l}\text { El profesor contribuye al cumplimiento de la misión institucional } \\
\text { fomentando en sus estudiantes una visión humanística, creativa y } \\
\text { emprendedora }\end{array}$ & .651 \\
\hline $\begin{array}{l}\text { El profesor manifiesta en su discurso y en la manera de llevar el curso, } \\
\text { dominio sobre el campo de conocimiento de su asignatura }\end{array}$ & .637 \\
\hline El profesor promueve la participación del estudiante & .628 \\
\hline $\begin{array}{l}\text { El profesor además de los parciales realizados tiene en cuenta otras } \\
\text { actividades realizadas por los estudiantes para la evaluación del curso }\end{array}$ & .612 \\
\hline El docente propicia el auto - aprendizaje de los estudiantes & .611 \\
\hline $\begin{array}{l}\text { El profesor contribuye a la generación de conocimiento y a la solución } \\
\text { de los problemas del entorno regional, nacional e internacional }\end{array}$ & .603 \\
\hline El profesor cumple responsablemente con el desarrollo del curso & .581 \\
\hline $\begin{array}{l}\text { El profesor conserva en su comunicación con el estudiante una } \\
\text { relación de mutuo respeto }\end{array}$ & .574 \\
\hline
\end{tabular}

Correlaciones intra-grupo combinadas entre las variables discriminantes y las funciones discriminantes canónicas tipificadas

Variables ordenadas por el tamaño de la correlación con la función.

Como se puede inferir, la variable que se encuentra más correlacionada con el modelo es si "El profesor facilita el aprendizaje de los estudiantes". Esta afirmación puede ser contrastada y fundamentada a partir de los nuevos planteamientos pedagógicos utilizados por la Institución a partir de la reflexión de los procesos de enseñanza - aprendizaje, en el cual estudiante debe ser parte activa en este proceso.

De la misma forma se puede establecer que para los estudiantes el aspecto que posee una menor importancia a la hora de calificar el desempeño general de sus docentes (pregunta No. 15) es si el profesor conserva en su comunicación una relación de mutuo respeto $(\mathrm{Co}=0.574)$.

En la Tabla No. 5 se pueden observar los coeficientes que acompañan a cada una de las preguntas y que guardan una estrecha relación con la correlación de cada una de las variables con la función discriminante general. 
Vásquez Rizo, F. E. y Gabalán Coello, J. (2006). Percepciones estudiantiles y su influencia en la evaluación del profesorado. Un caso en la Universidad Autónoma de Occidente, Cali-Colombia.

RELIEVE, v. 12, n. 2, p. 219-245. http://www.uv.es/RELIEVE/v12n2/RELIEVEv12n2_3.htm

TABLA No. 5

Coeficientes de las funciones canónicas discriminantes

\begin{tabular}{|c|c|}
\hline & Función \\
\hline & 1 \\
\hline El profesor cumple responsablemente con el desarrollo del curso & .162 \\
\hline $\begin{array}{l}\text { El profesor contribuye al cumplimiento de la misión institucional } \\
\text { fomentando en sus estudiantes una visión humanística, creativa y } \\
\text { emprendedora }\end{array}$ & .113 \\
\hline $\begin{array}{l}\text { El profesor contribuye a la generación de conocimiento y a la solución } \\
\text { de los problemas del entorno regional, nacional e internacional }\end{array}$ & .063 \\
\hline El profesor facilita el aprendizaje de los estudiantes & .297 \\
\hline El docente propicia el auto - aprendizaje de los estudiantes & .082 \\
\hline El profesor promueve la participación del estudiante & .113 \\
\hline $\begin{array}{l}\text { El profesor conserva en su comunicación con el estudiante una } \\
\text { relación de mutuo respeto }\end{array}$ & .140 \\
\hline $\begin{array}{l}\text { El profesor utiliza las evaluaciones como medio para mejorar el } \\
\text { aprendizaje }\end{array}$ & .161 \\
\hline $\begin{array}{l}\text { El profesor manifiesta en su discurso y en la manera de llevar el curso, } \\
\text { dominio sobre el campo de conocimiento de su asignatura }\end{array}$ & .228 \\
\hline $\begin{array}{l}\text { El profesor brinda orientaciones y alternativas claras y precisas a las } \\
\text { preguntas que se le formulan }\end{array}$ & .182 \\
\hline La propuesta de evaluación establecida es precisa y coherente & .121 \\
\hline $\begin{array}{l}\text { El profesor orienta y fomenta la autoevaluación por parte de los } \\
\text { estudiantes }\end{array}$ & .133 \\
\hline $\begin{array}{l}\text { El profesor además de los parciales realizados tiene en cuenta otras } \\
\text { actividades realizadas por los estudiantes para la evaluación del curso }\end{array}$ & .082 \\
\hline Las calificaciones asignadas por el profesor han sido justas & .288 \\
\hline (Constante) & -9.171 \\
\hline
\end{tabular}

Coeficientes no tipificados

Se puede evidenciar en el modelo que la más alta ponderación recae sobre las preguntas: 4, 14 y 9, que en su orden son: "El profesor facilita el aprendizaje de los estudiantes", "Las calificaciones asignadas por el profesor han sido justas" y "El profesor manifiesta en su discurso y en la manera de llevar el curso dominio sobre el campo".

El modelo definido para la percepción a partir del cuestionario de evaluación del desempeño docente, está determinado de la siguiente manera:

$$
\begin{array}{r}
\mathrm{D}=0.162(\mathrm{P} 1)+0.113(\mathrm{P} 2)+0.063(\mathrm{P} 3)+0.297(\mathrm{P} 4)+0.082(\mathrm{P} 5)+0.113(\mathrm{P} 6)+0.140(\mathrm{P} 7)+0.161(\mathrm{P} 8)+0.228 \\
(\mathrm{P} 9)+0.182(\mathrm{P} 10)+0.121(\mathrm{P} 11)+0.133(\mathrm{P} 12)+0.082(\mathrm{P} 13)+0.288(\mathrm{P} 14)-9.171
\end{array}
$$

En la Tabla No. 6 se realiza una prueba de hipótesis para la efectividad del modelo en el momento de discriminar. La hipótesis nula de dicha prueba consiste en afirmar que el modelo no es óptimo para discriminar, mientras que la hipótesis alterna argumenta lo contrario. 
Vásquez Rizo, F. E. y Gabalán Coello, J. (2006). Percepciones estudiantiles y su influencia en la evaluación del profesorado. Un caso en la Universidad Autónoma de Occidente, Cali-Colombia.

RELIEVE, v. 12, n. 2, p. 219-245. http://www.uv.es/RELIEVE/v12n2/RELIEVEv12n2 3.htm

TABLA No. 6

\section{Lambda de Wilks}

\begin{tabular}{|l|r|r|r|r|}
\hline $\begin{array}{l}\text { Contraste } \\
\text { las funciondde Wilks }\end{array}$ & hi-cuadradd & gl & Sig. \\
\hline 1 & .526 & 6125.430 & 14 & .000 \\
\hline
\end{tabular}

Se observa claramente que con una significancia de 0.000 (altamente significativo) se rechaza la hipótesis nula y por lo tanto el modelo escogido para la investigación es adecuado para la discriminación.

De igual manera, se realiza un contraste con la efectividad en la discriminación que tiene el modelo, de acuerdo al número de clasificaciones adecuadas.

Para la clasificación en la muestra seleccionada se lleva a cabo una partición, de la cual el $70 \%$ de los registros son utilizados para la muestra de análisis, es decir, aquella con la que se construye el modelo, y el $30 \%$ restante son utilizados para la muestra de validación.

Según el profesor Jorge de la Garza García (1995), "una de las consideraciones antes de derivar la ecuación discriminante consiste en partir la muestra en dos y utilizar para la etapa de derivación de $60 \%$ a $70 \%$ de los datos, y el restante $40 \%$ ó $30 \%$ se debe dejar para la etapa de validación del modelo, pues no es nada confiable el grado de error al pronosticar con los mismos datos que se derivaron de la ecuación, que si se usaran datos diferentes que en derivación".

En la muestra de análisis se clasifican correctamente el $90.7 \%$ de las percepciones de los estudiantes, mientras que en la de validación se clasifican correctamente el $90.4 \%$ de las percepciones. Esta situación permite verificar la estabilidad del modelo de clasificación.

Esto quiere decir, que en los dos escenarios (análisis y validación) se obtiene una clasificación adecuada por encima del $90 \%$ de las veces, situación que permite tener una efectividad en el proceso de clasificación de las observaciones. Como se puede ver en la Tabla No. 7.

\section{TABLA No. 7}

Resultados de la clasificaciórir,b

\begin{tabular}{|c|c|c|c|c|c|c|}
\hline & & & \multirow[b]{2}{*}{ Estándar Universidad } & \multicolumn{2}{|c|}{$\begin{array}{l}\text { Grupo de pertenencia } \\
\text { pronosticado }\end{array}$} & \multirow[b]{2}{*}{ Total } \\
\hline & & & & $\begin{array}{l}\text { Por encima } \\
\text { del estándar } \\
\text { de calidad }\end{array}$ & $\begin{array}{l}\text { Por debajo } \\
\text { del estándar } \\
\text { de calidad }\end{array}$ & \\
\hline \multirow[t]{4}{*}{$\begin{array}{l}\text { Casos } \\
\text { seleccionados }\end{array}$} & \multirow[t]{4}{*}{ Original } & Recuento & $\begin{array}{l}\text { Por encima del } \\
\text { estándar de calidad }\end{array}$ & 7433 & 733 & 8166 \\
\hline & & & $\begin{array}{l}\text { Por debajo del } \\
\text { estándar de calidad }\end{array}$ & 150 & 1214 & 1364 \\
\hline & & $\%$ & $\begin{array}{l}\text { Por encima del } \\
\text { estándar de calidad }\end{array}$ & 91.0 & 9.0 & 100.0 \\
\hline & & & $\begin{array}{l}\text { Por debajo del } \\
\text { estándar de calidad }\end{array}$ & 11.0 & 89.0 & 100.0 \\
\hline \multirow{4}{*}{\multicolumn{2}{|c|}{$\begin{array}{l}\text { Casos no } \\
\text { seleccionados }\end{array}$}} & Recuento & $\begin{array}{l}\text { Por encima del } \\
\text { estándar de calidad }\end{array}$ & 3129 & 329 & 3458 \\
\hline & & & $\begin{array}{l}\text { Por debajo del } \\
\text { estándar de calidad }\end{array}$ & 62 & 548 & 610 \\
\hline & & \multirow[t]{2}{*}{$\%$} & $\begin{array}{l}\text { Por encima del } \\
\text { estándar de calidad }\end{array}$ & 90.5 & 9.5 & 100.0 \\
\hline & & & $\begin{array}{l}\text { Por debajo del } \\
\text { estándar de calidad }\end{array}$ & 10.2 & 89.8 & 100.0 \\
\hline
\end{tabular}

a. Clasificados correctamente el $90.7 \%$ de los casos agrupados originales seleccionados.

b. Clasificados correctamente el $90.4 \%$ de casos agrupados originales no seleccionados. 
Vásquez Rizo, F. E. y Gabalán Coello, J. (2006). Percepciones estudiantiles y su influencia en la evaluación del profesorado. Un caso en la Universidad Autónoma de Occidente, Cali-Colombia.

RELIEVE, v. 12, n. 2, p. 219-245. http://www.uv.es/RELIEVE/v12n2/RELIEVEv12n2 3.htm

\section{CONCLUSIONES}

Al comparar la percepción de los estudiantes se concluye que existe similitud entre opiniones intra e inter Programas, lo que determina perfiles de estudiantes y de qué manera reaccionan en el momento de emitir juicios valorativos de sus docentes.

Aproximadamente la mitad de la variación en la calificación general que hacen los estudiantes sobre el desempeño de sus docentes (pregunta No. 15) es explicada por la variación de cada uno de los ítems del cuestionario (preguntas Nos. 1 a 14), lo que implica que existen factores externos que no se tiene en cuenta en el cuestionario y que influyen en los estudiantes en el momento de emitir juicios sobre sus profesores. Con base en el análisis cualitativo se determinan los siguientes:

- "El nivel de empatía que desarrolla el profesor con los estudiantes, reflejado en relaciones amistosas, aspectos personales y humanos del docente".

- "El número de docentes que se hayan tenido a lo largo de la carrera, como elemento de comparación".

- "La experiencia que ha tenido el profesor en el sector empresarial".

- "La complejidad de la asignatura".

- "La capacidad de aprendizaje de algunos estudiantes, en cuanto a las diferencias de opinión que pueden presentar los estudiantes aventajados y los rezagados".

Se establece que las cinco preguntas del cuestionario que más influyen en los estudiantes para calificar de manera general al profesor son:

- "El profesor facilita el aprendizaje de los estudiantes".

- "Las calificaciones asignadas por el profesor han sido justas".

- "El profesor brinda orientaciones y alternativas claras y precisas a las preguntas que se le formulan".

- "El profesor orienta y fomenta la autoevaluación por parte de los estudiantes".
- "El profesor utiliza las evaluaciones como medio para mejorar el aprendizaje".

El estudio arroja que el aspecto que menos tienen en cuenta los estudiantes de la Universidad Autónoma de Occidente para calificar el desempeño general de sus docentes, es el relacionado con la comunicación entre los actores del aula de clases.

A partir del análisis cuantitativo se determina un modelo que establece el grado de relevancia de las variables en la percepción final del estudiante. En el cualitativo se determinan algunos elementos que forman parte de la visión estudiantil sobre el desempeño de sus docentes, en los cuales se involucra la noción de calidad y lo que esto representa para los estudiantes.

A nivel de los diferentes Programas y Facultades que constituyen la Universidad Autónoma de Occidente se pueden establecer conclusiones particulares tales como:

- En la Facultad de Ciencias Económicas y Administrativas, los factores más relevantes son: "El profesor facilita el aprendizaje de los estudiantes" y "Las calificaciones asignadas por el profesor han sido justas".

- En el Programa de Administración de Empresas se observa que a medida que existe una mejor calificación en el ítem "La propuesta de evaluación establecida es precisa y coherente" se presenta una mayor tendencia a emitir un juicio valorativo negativo general del docente.

- En dos de los Programas de la Facultad de Comunicación Social (Comunicación Social - Periodismo y Diseño de la Comunicación Gráfica) la variable más relevante es "El profesor manifiesta en su discurso y en la manera de llevar el curso, dominio sobre el campo de conocimiento de su asignatura".

- Los estudiantes del Programa de Comunicación Publicitaria asignan una mayor importancia a la pregunta "El profesor conserva en su comunicación con el estudiante una relación de mutuo respeto". 
Vásquez Rizo, F. E. y Gabalán Coello, J. (2006). Percepciones estudiantiles y su influencia en la evaluación del profesorado. Un caso en la Universidad Autónoma de Occidente, Cali-Colombia.

RELIEVE, v. 12, n. 2, p. 219-245. http://www.uv.es/RELIEVE/v12n2/RELIEVEv12n2 3.htm

- Si el profesor además de los parciales tiene en cuenta otras actividades para evaluar el curso es muy probable que en el desempeño general sea mal calificado, según los estudiantes del Programa de Diseño de la Comunicación Gráfica.

- En el Programa de Administración del Medio Ambiente y de los Recursos Naturales no existe mayor relación entre si el profesor propicia el autoaprendizaje en los estudiantes con la calificación general de su desempeño.

- Un elemento común en la Facultad de Ingeniería, y que tiene gran importancia en el momento de definir el desempeño general del profesor es si este facilita el aprendizaje de los estudiantes.

- En el Programa de Ingeniería de Producción existe una relación inversamente proporcional entre la variable relacionada con la justicia en las calificaciones asignadas por el profesor y la calificación general del mismo. Igual sucede con la variable "El profesor brinda orientaciones y alternativas claras y precisas a las preguntas que se le formulan" en el Programa de Ingeniería Informática.

- Para los estudiantes pertenecientes a los Primeros Semestres, la pregunta que más peso tiene en la percepción final sobre el profesor es la relacionada con la justicia en las calificaciones asignadas por el profesor.

- Llama mucho la atención que, en los estudiantes de Primeros Semestres, aproximadamente el $40 \%$ de la variación en la opinión general que se tiene del docente depende de los aspectos evaluados en el cuestionario, lo que indica claramente que influyen de manera considerable aspectos externos, explicados en párrafos anteriores.

El estudio permite realizar las siguientes recomendaciones:

-No a todos los estudiantes se les puede interrogar sobre todos los aspectos del trabajo de sus profesores, debido a que la subjetividad y las relaciones de amistad o empatía juegan un factor decisivo en el momento de evaluar a los docentes, como se pudo establecer a través de los focus group (práctica cualitativa).

-Con respecto a sí valdría la pena presentar cuestionarios de opinión diferentes para estudiantes, dependiendo del semestre en que se encuentren, es clara la división que se hace en la investigación, desde el punto de vista metodológico, al conformar dos grupos de análisis: estudiantes de Primeros Semestres (1-3) y estudiantes de semestres superiores (4-10). Esta división permite establecer que influye de manera relevante el semestre en que se encuentre el estudiante, debido a que los del primer grupo cuentan con menos elementos de juicio, como ellos mismos lo definen, y no se encuentran familiarizados con el proceso de evaluación.

-Es fundamental contrastar información de rendimiento de los estudiantes con la información obtenida de sus percepciones acerca del trabajo del profesor, ya que muchas veces los estudiantes de bajo rendimiento académico tienden a delegar la responsabilidad de su desempeño en las prácticas pedagógicas de sus docentes. Esto se resume en la frase escuchada muchas veces "si me califica mal yo lo califico mal". El anterior comentario explica además que algunas percepciones sobre el profesor se encuentran condicionadas por el rendimiento del estudiantado; es decir, pocas veces se asume un papel responsable con respecto al proceso de enseñanza, por parte del estudiante.

\section{7.- BIBLIOGRAFÍA}

Arnaz, J. A. y Yuren, T. (1994). Docencia. En Serie Documentos. Mexicali: CETYS.

De La Garza García, J. (1995). Análisis discriminante. En Análisis de la Información Mercadológica a través de la Estadística Multivariante: Capítulo 4. Primera Edición. Ciudad de México: Alambra.

Deming, E. (1998). Fuera de la crisis. Massachussets: MIT. 
Vásquez Rizo, F. E. y Gabalán Coello, J. (2006). Percepciones estudiantiles y su influencia en la evaluación del profesorado. Un caso en la Universidad Autónoma de Occidente, Cali-Colombia.

RELIEVE, v. 12, n. 2, p. 219-245. http://www.uv.es/RELIEVE/v12n2/RELIEVEv12n2 3.htm

Díaz, L. G. (2002) Análisis discriminante. En Estadística Multivariada: Inferencia y Métodos: Capítulo 8. Bogotá: Universidad Nacional de Colombia.

Díaz, L. G. (2002). Análisis estadístico de datos categóricos. En Notas de Clase del Departamento de Estadística de la Universidad Nacional de Colombia. Bogotá: Universidad Nacional de Colombia.

Etxegaray, F. y Sanz, I. (1991). La evaluación de la docencia del profesor universitario. San Sebastián: Universidad del País Vasco.

García Carrasco, J. y Villa Sánchez, A. (1984). Líneas dominantes de investigación en el análisis de la función docente. Madrid. Instituto Colombiano para el Fomento de la Educación Superior (2000). Aprender a Investigar. En Serie ICFES. Bogotá: ICFES.

Marsh, H. W. (1982). Validity of student's evaluations of college teaching a multirate. Multimethod Analyse. En Journal of Educational Psychology. Vol. 74. No. 2. Washington D.C.: American Psychological Association.

Mayor Ruiz, C. (1996). Las funciones del profesor universitario analizadas por sus protagonistas. Un estudio atendiendo al grupo de titulación y los años de experiencia, en la Universidad de Sevilla. En Revista Electrónica de Investigación y Evaluación
Educativa. Vol. 2. No. 1. Consultado en http://www.uv.es/RELIEVE/v2n1/RELIEV Ev2n1.htm el 10 de Febrero de 2006.

Meade, D. (1996). El profesor de calidad. Monterrey: Instituto Tecnológico de Educación y Estudios Superiores de Monterrey ITESM.

Moreno García, J. M. (1972). Evaluación de los factores que deciden la eficacia didáctica del profesorado. En Bordón. No. 187. Madrid: Sociedad Española de Pedagogía.

Patton, M. Q. (1987). How to use qualitative methods in evaluation. Newbury Park, California: SAGE Publications Inc.

Peña, D. (2002). Análisis de datos multivariante. $1 \mathrm{Ed}$. Universidad Carlos III de Madrid. Madrid: McGraw Hill.

Rizo Moreno, H. E. (1999). Evaluación del docente universitario. En Revista Electrónica Interuniversitaria de Formación del Profesorado. Vol. 2 No. 1. Zaragoza: Asociación Universitaria de Formación del Profesorado - AUFOP.

Rizo Moreno, H. E. (2004). Evaluación del profesor universitario. Una visión institucional. En Revista Iberoamericana de Educación. No. 34.

Universidad Autónoma de Occidente (2002). Reformando el Presente con Visión de Futuro. Documento de Rectoría. Cali: UAO.

\section{ANEXO 1}

UNIVERSIDAD AUTÓNOMA DE OCCIDENTE

CENTRO DE DESARROLLO ACADÉMICO

EVALUACIÓN DEL DESEMPEÑO DOCENTE

CUESTIONARIO DE OPINIÓN PARA ALUMNOS 
Vásquez Rizo, F. E. y Gabalán Coello, J. (2006). Percepciones estudiantiles y su influencia en la evaluación del profesorado. Un caso en la Universidad Autónoma de Occidente, Cali-Colombia.

RELIEVE, v. 12, n. 2, p. 219-245. http://www.uv.es/RELIEVE/v12n2/RELIEVEv12n2 3.htm

OBJETIVO: Obtener información confiable que permita evaluar el desarrollo de los cursos, con el fin de definir estrategias conducentes al mejoramiento continuo de los procesos docentes en la Institución.

\section{INSTRUCCIONES}

- Lea cuidadosamente cada enunciado antes de marcar su respuesta.

- Usted encontrará una afirmación y unos referentes para su respuesta.

- Es fundamental que sus respuestas sean el resultado de un análisis crítico y objetivo de la situación que se le plantea.

- Califique en una escala de 1 a 5 en cada una de las situaciones planteadas de acuerdo con los referentes de cada pregunta. 1 es calificación muy mala y se asigna cuando el docente no cumple con ninguno de los referentes, $\mathbf{5}$ es una calificación excelente y se asigna cuando el profesor cumple cabalmente con todos los referentes.

\section{CUESTIONARIO}

\section{RESPONSABILIDAD Y COMPROMISO INSTITUCIONAL}

1. El profesor cumple responsablemente con el desarrollo del curso cuando:

- Establece y cumple las condiciones relacionadas con: objetivos, metodología y forma de evaluar el curso.

- Asiste a todas las clases.

- Empieza y termina su clase a tiempo.

2. El profesor contribuye al cumplimiento de la misión institucional fomentando en sus estudiantes una visión humanística, creativa y emprendedora cuando:

- Ejemplifica con sus actitudes y expresiones una manera apropiada de relacionarse humanamente con sus semejantes.

- Incentiva la originalidad en las realizaciones de los estudiantes.

- Motiva a que los alumnos agreguen valor a sus productos y le da sentido práctico y de utilidad social a las temáticas desarrolladas en clases.

3. El profesor contribuye a la generación de conocimiento y a la solución de los problemas del entorno regional, nacional e internacional cuando:

- Fomenta actitudes investigativas en los estudiantes.

- Genera espacios de reflexión acerca de los beneficios que traería la aplicación de los conceptos o técnicas estudiadas para la comunidad.

- Posibilita el debate frente a las implicaciones éticas de los desarrollos científicos, culturales, tecnológicos, políticos, sociales y económicos estudiados.

\section{METODOLOGIA.}

4. El profesor facilita el aprendizaje de los estudiantes cuando:

- Expresa los conceptos de forma coherente y ordenada.

- Expone conceptos con claridad y precisión. 
Vásquez Rizo, F. E. y Gabalán Coello, J. (2006). Percepciones estudiantiles y su influencia en la evaluación del profesorado. Un caso en la Universidad Autónoma de Occidente, Cali-Colombia.

RELIEVE, v. 12, n. 2, p. 219-245. http://www.uv.es/RELIEVE/v12n2/RELIEVEv12n2 3.htm

- Se apoya en las exposiciones o trabajos de los estudiantes para aclarar y profundizar conceptos con el grupo.

5. El docente propicia el auto-aprendizaje de los estudiantes cuando:

- Genera estrategias que inducen a los estudiantes a realizar consultas bibliográficas y a utilizar diferentes medios de información.

- Incorpora al curso actividades que permiten a los estudiantes obtener planteamientos y resultados propios.

6. El profesor promueve la participación del estudiante cuando:

- Plantea actividades que permiten al alumno expresar e intercambiar ideas, opiniones y experiencias con sus compañeros.

- Crea un ambiente de confianza y respeto en el que los alumnos pueden opinar libremente.

- Utiliza técnicas que favorecen el debate, la discusión y las propuestas.

7. El profesor conserva en su comunicación con el estudiante una relación de mutuo respeto cuando:

- Se comunica con ellos en forma respetuosa y cordial y propicia que los estudiantes se dirijan a él en la misma forma.

- Admite en los alumnos posiciones divergentes sustentadas en el desarrollo del curso.

- Respeta y hace respetar los compromisos y normas establecidos para el desarrollo del curso (formas de evaluación, desarrollo de trabajos, etc.).

8. El profesor utiliza las evaluaciones como medio para mejorar el aprendizaje cuando:

- Suministra al estudiante información oportuna acerca de los trabajos, tareas, exámenes, ejercicios, etc., que el estudiante le presente.

- Propicia la identificación de las fortalezas y debilidades del estudiante durante el desarrollo del curso.

\section{DOMINIO ASIGNATURA}

9. El profesor manifiesta en su discurso y en la manera de llevar el curso, dominio sobre el campo de conocimiento de su asignatura cuando:

- Muestra seguridad sobre las temáticas expuestas en clase.

- Enriquece el curso con información y bibliografía actualizada.

- Retroalimenta adecuadamente a los distintos planteamientos analizados en clase.

10. El profesor brinda orientaciones y alternativas claras y precisas a las preguntas que se le formulan cuando:

- Sugiere estrategias para la búsqueda de soluciones.

- Ubica y dimensiona los planteamientos o interrogantes de sus estudiantes.

- Se involucra en la búsqueda de soluciones y caminos alternativos cuando no tiene la respuesta precisa para una inquietud o interrogante.

- Las respuestas que proporciona, en la mayoría de los casos, resuelven adecuadamente las preguntas formuladas.

\section{EVALUACION}

11. La propuesta de evaluación establecida es precisa y coherente cuando:

- Presenta con claridad y antelación los criterios de evaluación.

- Utiliza técnicas e instrumentos adecuados para evaluar el logro de los objetivos propuestos.

- Los elementos involucrados en la evaluación son acordes con los contenidos propuestos. 
Vásquez Rizo, F. E. y Gabalán Coello, J. (2006). Percepciones estudiantiles y su influencia en la evaluación del profesorado. Un caso en la Universidad Autónoma de Occidente, Cali-Colombia.

RELIEVE, v. 12, n. 2, p. 219-245. http://www.uv.es/RELIEVE/v12n2/RELIEVEv12n2 3.htm

12. El profesor orienta y fomenta la autoevaluación por parte de los estudiantes cuando:

- Estimula la participación de los alumnos en la evaluación de su propio aprendizaje.

- Promueve en el estudiante la reflexión sobre la forma como desarrolla sus actividades de aprendizaje y los resultados obtenidos.

- Motiva acciones concretas para su mejoramiento.

13. El profesor además de los parciales realizados tiene en cuenta otras actividades realizadas por los estudiantes para la evaluación del curso cuando:

- Realiza actividades que comprometen el esfuerzo de los estudiantes y las tiene en cuenta en su propuesta evaluativa.

- Establece alguna ponderación en la calificación final para actividades que se diferencian claramente de las pruebas escritas.

14. Las calificaciones asignadas por el profesor han sido justas cuando:

- Los trabajos, tareas y exámenes están bien diseñados, son relevantes y con el adecuado nivel de exigencia.

- Usa el mismo criterio para evaluar a todos los alumnos.

- Los criterios con que se asignan son claros y bien aplicados.

\section{OPINION GENERAL}

\section{En qeneral y de acuerdo a su desempeño, indique la calificación que le asigna al profesor.}

\section{NOTAS}

[1] La revisión de los procedimientos estadísticos ha sido realizada por los Estadísticos Daiver Cardona Salgado, Director del Consultorio Estadístico y Otoniel Madrid, catedrático de la asignatura "Análisis de Datos Multivariados", profesores del Departamento de Matemáticas de la Universidad Autónoma de Occidente.

[2] Para interpretar el coeficiente de correlación, Colton ha establecido los siguientes lineamientos generales:

- Valor de $\mathrm{r}$ de 0 a 0.25 implica que no existe correlación entre ambas variables.
- Valor de $\mathrm{r}$ de 0.25 a 0.50 implica una correlación de baja a moderada.

- Valor de $\mathrm{r}$ de 0.50 a 0.75 implica correlación de moderada a buena.

- Valor de $\mathrm{r}$ de 0.75 o mayor, implica una muy buena a excelente correlación.

- Estos rangos de valores se pueden extrapolar a correlaciones negativas también.

[3] En este caso la correlación canónica, al elevarla al cuadrado, indica qué porcentaje de la variabilidad, de la pregunta No. 15 , es explicado por la variabilidad de las preguntas anteriores. 
Vásquez Rizo, F. E. y Gabalán Coello, J. (2006). Percepciones estudiantiles y su influencia en la evaluación del profesorado. Un caso en la Universidad Autónoma de Occidente, Cali-Colombia.

RELIEVE, v. 12, n. 2, p. 219-245. http://www.uv.es/RELIEVE/v12n2/RELIEVEv12n2 3.htm

\section{ABOUT THE AUTHORS / SOBRE LOS AUTORES}

Fredy Eduardo Vásquez Rizo (fvasquez@uao.edu.co). Comunicador Social - Periodista, integrante del Grupo de Investigación en Educación y del Grupo de Investigación en Gestión del Conocimiento y Sociedad de la Información. Sus áreas de actuación son Educación, Investigación, Sistemas de Información, y Gestión del Conocimiento. Estudia actualmente la Maestría en Ciencias de la Información y Administración del Conocimiento en el ITESM de México Su dirección postal es: Universidad Autónoma de Occidente, Calle 25 No. 115 - 85 Km 2 vía Cali - Jamundí. Santiago de Cali, Colombia.

Jesús Gabalán Coello (jgabalan@uao.edu.co). Ingeniero Industrial, integrante del Grupo de Investigación en Educación. Actualmente es Analista de la Oficina de Planeación y Desarrollo, adscrita a Rectoría, de la Universidad Autónoma de Occidente. Sus áreas de actuación son Estadística Aplicada a la Educación, Garantía de Control de Calidad e Indicadores de Gestión. Es profesor de Estadística y se encuentra desarrollando la Maestría en Ingeniería con énfasis en Ingeniería Industrial, en la Universidad del Valle, Colombia. Su dirección postal es: Universidad Autónoma de Occidente, Calle 25 No. 115 - 85 Km 2 vía Cali - Jamundí. Santiago de Cali, Colombia. 
Vásquez Rizo, F. E. y Gabalán Coello, J. (2006). Percepciones estudiantiles y su influencia en la evaluación del profesorado. Un caso en la Universidad Autónoma de Occidente, Cali-Colombia.

RELIEVE, v. 12, n. 2, p. 219-245. http://www.uv.es/RELIEVE/v12n2/RELIEVEv12n2 3.htm

\section{ARTICLE RECORD / FICHA DEL ARTÍCULO}

\begin{tabular}{|l|l|l|l}
\hline $\begin{array}{l}\text { Reference / } \\
\text { Referencia }\end{array}$ & $\begin{array}{l}\text { Vásquez Rizo, Fredy Eduardo y Gabalán Coello, Jesús (2006). Percepciones estudiantiles y su influen } \\
\text { en la evaluación del profesorado. Un caso en la Universidad Autónoma de Occidente, Cali - Colombia. } \\
\text { fecha). }\end{array}$ \\
\hline $\begin{array}{l}\text { Title / Títu- } \\
\text { lo }\end{array}$ & $\begin{array}{l}\text { Percepciones estudiantiles y su influencia en la evaluación del profesorado. Un caso en la Universidad } \\
\text { Autónoma de Occidente, Cali - Colombia. [Student perceptions and their influence on the evaluation } \\
\text { the teaching staff. A case in Universidad Autonoma de Occidente, Cali - Colombia] }\end{array}$ \\
\hline $\begin{array}{l}\text { Authors / } \\
\text { Autores }\end{array}$ & $\begin{array}{l}\text { Fredy Eduardo Vásquez Rizo. Jesús Gabalán Coello } \\
\text { Review / } \\
\text { Revista }\end{array}$ & Revista ELectrónica de Investigación y EValuación Educativa (RELIEVE), v. 12, n. 2 \\
\hline \begin{tabular}{l} 
ISSN \\
\hline $\begin{array}{l}\text { Publication } \\
\text { date / } \\
\text { Fecha de } \\
\text { publicación }\end{array}$
\end{tabular} & $\begin{array}{l}\text { 1134-4032 } \\
\text { July 27) }\end{array}$ \\
\hline
\end{tabular}

The investigation results are presented: "An investigation or research about the most relevant variables from the student's point of view which influence the general opinion about the teacher's performance in the Universidad Autonoma de Occidente". The problems about the influence from the students perception in evaluating their teachers is taken on from two complementary perspectives: The first constitutes the qualitative analysis which has as a support the group interviews realized in each one of the academic programs. The second involves the quantitative analysis based on multivariate statistic tools. These perspectives allow elaborating a model to establish the grade of relevance each one of the items used to evaluate the teacher has, in relation with the general grading that is assigned to his/her performance. Finally, the most important aspects are evidenced for the students at the moment of stating a valuable judgment over their teachers

Abstract / Se presentan los resultados de la investigación: "Estudio sobre las variables más relevantes, desde el punto de vista estudiantil, que influyen en la opinión general sobre el desempeño de los docentes en la Universidad Autónoma de Occidente”. Se aborda la problemática sobre la influencia que tienen las percepciones estudiantiles en la evaluación del profesorado desde dos perspectivas complementarias: La primera de ellas constituye el análisis cualitativo, que tiene como soporte entrevistas grupales llevadas a cabo con estudiantes de cada uno de los Programas Académicos. La segunda involucra el análisis cuantitativo, basándose en herramientas de estadística multivariada. Estas perspectivas permiten elaborar un modelo que establece el grado de relevancia que tiene cada uno de los ítems a través de los cuales se evalúa un profesor, en relación con la calificación general que se asigna a su desempeño. Finalmente, se evidencia qué aspectos son los más importantes, para los estudiantes, en el momento de emitir un juicio valorativo sobre sus docentes.

Student Perceptions, Evaluation, Quantitative Analysis, Qualitative Analysis, Teacher Performance, University

Keywords

Percepciones Estudiantiles, Evaluación, Análisis Cuantitativo, Análisis Cualitativo, Desempeño Docente, Universidad

Institution /

Institución

Universidad Autónoma de Occidente (Colombia)

Publication

site /

$\underline{\text { http://www.uv.es/RELIEVE }}$

\section{Dirección}

Language /

Idioma

Spanish (Title, abstract and keywords in English ) 
Vásquez Rizo, F. E. y Gabalán Coello, J. (2006). Percepciones estudiantiles y su influencia en la evaluación del profesorado. Un caso en la Universidad Autónoma de Occidente, Cali-Colombia.

RELIEVE, v. 12, n. 2, p. 219-245. http://www.uv.es/RELIEVE/v12n2/RELIEVEv12n2 3.htm

\section{Revista ELectrónica de Investigación y $\mathbf{E V}$ aluación Educativa (RELIEVE)}

\section{[ ISSN: 1134-4032 ]}

(C) Copyright, RELIEVE. Reproduction and distribution of this articles it is authorized if the content is no modified and their origin is indicated (RELIEVE Journal, volume, number and electronic address of the document).

(C) Copyright, RELIEVE. Se autoriza la reproducción y distribución de este artículo siempre que no se modifique el contenido y se indique su origen (RELIEVE, volumen, número y dirección electrónica del documento). 